Progress in Nuclear Science and Technology

Volume 5 (2018) pp.157-160

\title{
ARTICLE
}

\section{Crystal structure and magnetic properties of new ternary uranium compound $\mathrm{U}_{3} \mathrm{TiBi}_{9}$}

\author{
Gaku Motoyama $^{a^{*}}$, Yoshinori Haga ${ }^{\mathrm{b}}$, Akira Yamaguchic, Ikuto Kawasakic, \\ Akihiko Sumiyama ${ }^{c}$ and Tomoo Yamamura ${ }^{\mathrm{d}}$ \\ ${ }^{a}$ Graduate School of Material Science, Shimane University, 1060 Nishikawatsu, Matsue, Shimane, 690-8504, Japan; ${ }^{b}$ Advanced \\ Science Research Center, Japan Atomic Energy Agency, Tokai, Naka-gun, Ibaraki, 319-1195, Japan; ${ }^{c}$ Graduate School of \\ Material Science, University of Hyogo, Kouto, Ako-gun, Hyogo, 672-8504, Japan; ${ }^{d}$ Institute for Materials Research, Tohoku \\ University, Aoba-ku, Sendai, Miyagi, 980-8577, Japan
}

The crystal structure of the newly discovered compound $\mathrm{U}_{3} \mathrm{TiBi}_{9}$ was determined from single-crystal X-ray diffraction data. The space group is $P 6_{3} / m$ with the lattice parameters $a=11.691 \AA, c=6.331 \AA$. $\mathrm{U}_{3} \mathrm{TiBi}_{9}$ shows an antiferromagnetic ordering at $T_{\mathrm{N}}=31.5 \mathrm{~K}$. A clear anomaly was observed at $T_{\mathrm{N}}$ on the temperature dependences of magnetic susceptibility and electrical resistivity.

Keywords: uranium intermetallic; inversion symmetry; antiferromagnetic ordering; $U_{3} T_{i B i_{9}}$

\section{Introduction}

The uranium compounds exhibit a variety of superconductivities and magnetic behaviors including Pauli itinerant, heavy fermion and local moment ordering, where the $5 \mathrm{f}$ electrons play principal roles [1-3]. The heavy fermion originates from the hybridization effect between the conduction electrons and the $f$ electrons. The $5 f$ electrons have an intermediate character between the localized $4 \mathrm{f}$ electrons of rare-earth compounds and the itinerant $3 \mathrm{~d}$ electrons of transition metals. This characteristic of the $5 \mathrm{f}$ electrons leads to the variety of physical properties of the $U$ compounds.

The crystallographical studies of the ternary Ce-Ti-Sb and U-Ti-Sb systems have already been carried out by $\mathrm{S}$. H. D. Moore et al. [4] and A. Mar et al. [5], respectively. The existence of some ternary compounds including $\mathrm{Ce}_{3} \mathrm{TiSb}_{5}, \mathrm{CeTi}_{3} \mathrm{Sb}_{4}$ and $\mathrm{U}_{3} \mathrm{TiSb}_{5}$ was already confirmed. However there has been no report of the ternary compound in the Ce-Ti-Bi and U-Ti-Bi systems. As the results of our investigation in the $\mathrm{Ce}-\mathrm{Ti}-\mathrm{Bi}$ system, we found two new compounds $\mathrm{Ce}_{3} \mathrm{TiBi}_{5}$ and $\mathrm{CeTi}_{3} \mathrm{Bi}_{4}$, which are obtained by a Bi self-flux method, and their single crystals form needles and plates, respectively [6]. Each of the two new compounds is isostructural with the antimonides $\mathrm{Ce}_{3} \mathrm{TiSb}_{5}\left(\mathrm{~Pb}_{3} / \mathrm{mcm}\right)$ and $\mathrm{CeTi}_{3} \mathrm{Sb}_{4}(\mathrm{Fmmm})$. It is found that $\mathrm{Ce}_{3} \mathrm{TiBi}_{5}$ and $\mathrm{CeTi}_{3} \mathrm{Bi}_{4}$ exhibit an antiferromagnetic ordering at $3.5 \mathrm{~K}$ and $5.0 \mathrm{~K}$, respectively. In this paper, we report the discovery of the first U-Ti-Bi intermetallic compound and its magnetic properties.

*Corresponding author. Email: motoyama@riko.shimane-u.ac.jp

\section{Experimental}

The single crystal samples were prepared by the $\mathrm{Bi}$ self-flux method. The purities of the materials of $\mathrm{U}, \mathrm{Ti}$ and $\mathrm{Bi}$ are 99.9, 99.9 and $99.99 \%$, respectively. The starting materials were placed in the ratio $\mathrm{U}: \mathrm{Ti}: \mathrm{Bi}=$ $3: 1: 40$ into an alumina crucible and sealed under high vacuum in a quartz tube. The sealed ampoule was heated up to $1000{ }^{\circ} \mathrm{C}$, kept for $11 \mathrm{~h}$, followed by a slow cool at $2{ }^{\circ} \mathrm{C} / \mathrm{h}$ to $500{ }^{\circ} \mathrm{C}$. The excess bismuth flux was removed from the crystals by using a centrifuge, then the needleshaped single crystals were obtained. The prepared single crystals of the habit of $\mathrm{U}_{3} \mathrm{TiBi}_{9}$ are shown in Figure 1. The crystal was easily damaged in air, and collapses during the first several hours after leaving it in air.

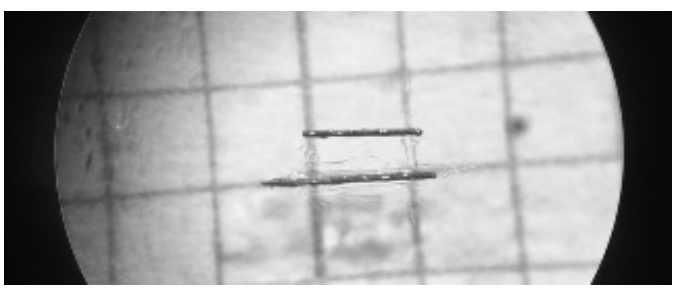

Figure 1. Single crystals of $\mathrm{U}_{3} \mathrm{TiBi}_{9}$. The needle axis corresponds to the crystallographic $\mathrm{c}$ axis. A grid of the cross-section is equal to $1 \times 1 \mathrm{~mm}^{2}$. The weight of the largest crystal, which was obtained in this work, is $0.07(3) \mathrm{mg}$.

Crystal structure investigation was done by single-crystal X-ray diffraction experiments. Diffraction data were collected using an imaging plate detector with $\mathrm{Mo} \mathrm{K} \alpha$ radiation. The single crystal sample for the 
X-ray diffraction was coated by vacuum grease to avoid oxidization and no apparent change in the diffraction intensities has been observed during the measurement. Structural solution by the direct method and refinements of the structural parameters were performed using the SHELX software [7].

Electrical resistivity was measured by a standard four-terminal method, and magnetic susceptibility and magnetization were measured using a commercial SQUID magnetometer (Quantum Design MPMS). To prevent the sample from oxidizing, the resistivity measurement was carried out in the pressure cell filled with Daphne7373 as pressure transmedium. The applied pressure was almost ambient pressure.

\section{Crystal structure}

The crystal structure has been successfully solved by the single crystal X-ray diffraction. 7757 Bragg reflections were successfully indexed for the hexagonal structure $\left(P 6_{3} / \mathrm{m}\right)$ with lattice parameters $a=11.691(1)$ $\AA$ and $c=6.331(1) \AA$, where 791 were unique. The first attempt by reference to the structure of the $\mathrm{Ce}_{3} \mathrm{TiBi}_{5}$ gave only a poor agreement between the calculated and observed intensities. The space-group $P 6_{3} / m$ of the obtained needle-shape crystals is different from that of $\mathrm{Ce}_{3} \mathrm{TiBi}_{5}$, although the crystal shape is very similar between the obtained crystal and $\mathrm{Ce}_{3} \mathrm{TiBi}_{5}$. And then $a$ is larger than that of $\mathrm{Ce}_{3} \mathrm{TiBi}_{5}$, although $c$ is smaller [6]. Lattice parameters of a U-compound usually shrink in comparison with the isostructural Ce-compound. This unexpected results derive from a change from the single $\mathrm{Bi}$-chain to triangle prism of $\mathrm{Bi}$ which lies between the three $\mathrm{TiBi}_{6}$ octahedron, as shown in Figure 2. The octahedral structure is common structure between the both compounds. As the result, the chemical composition of the obtained crystal is not $\mathrm{U}_{3} \mathrm{TiBi}_{5}$ but $\mathrm{U}_{3} \mathrm{TiBi}_{9}$. Moreover the change additionally results in a
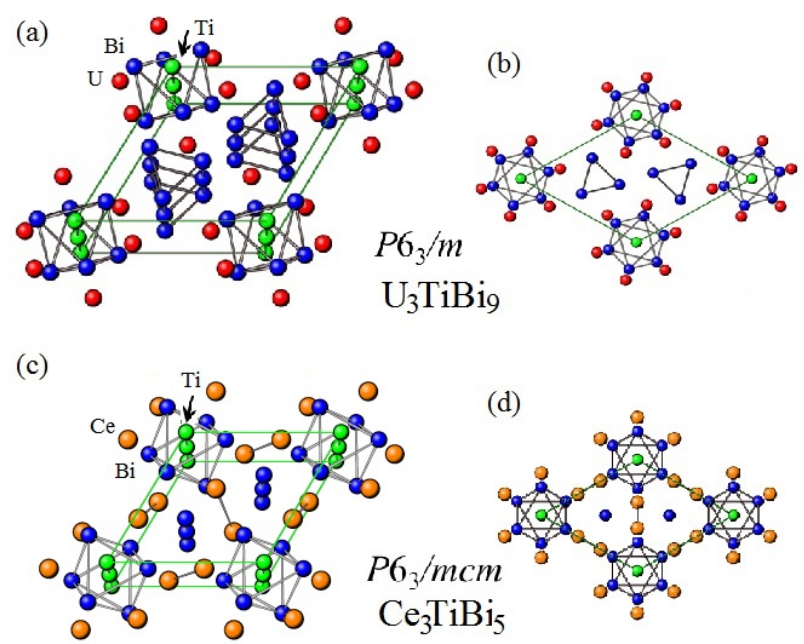

Figure 2. Comparison between crystal structures of $\mathrm{U}_{3} \mathrm{TiBi}_{9}(\mathrm{a}$, b) and $\mathrm{Ce}_{3} \mathrm{TiBi}_{5}(\mathrm{c}, \mathrm{d})$. slight octahedron's rotation about the c-axis. The crystallographic parameters of $\mathrm{U}_{3} \mathrm{TiBi}_{9}$ as well as the refinement details are shown in Table 1. The good agreement between the observed and calculated structure factors assures validity of the structural model.

In the crystal structure of $\mathrm{Ce}_{3} \mathrm{TiBi}_{5}$, the $\mathrm{Ce}$ atoms are located in the face of ac-plane and form zig-zag chains along parallel to the c-axis. However, because of the slight rotation of the octahedron, the $U$ atoms in the crystal structure of $\mathrm{U}_{3} \mathrm{TiBi}_{9}$ are off from the face of the ac-plane. The distance between the $U$ atoms get longer and the $U$ atoms no longer form a zig-zag chain, unfortunately. The nearest U-U distance is $4.774 \AA$, which is decided from the distance between the two $U$ atoms surrounded the same Bi octahedron.

Table 1. Crystallographic parameters for $\mathrm{U}_{3} \mathrm{TiBi}_{9}$ (space group: $P 6_{3} / m$; lattice parameters $a=11.691(1), c=6.331(1) \AA$ ). The data were corrected for Lorentz and polarization effects. The overall agreement factor $R_{1}=\sum|| F_{\mathrm{o}}|-| F_{\mathrm{c}}|| \sum\left|F_{\mathrm{o}}\right|=0.0591$, where $F_{\mathrm{o}}$ and $F_{\mathrm{c}}$ are observed and calculated structure factors, where anisotropic atomic displacement parameters have been refined.

\begin{tabular}{cccccc}
\hline Atom & Site & sym. & $x$ & $y$ & $z$ \\
\hline Bi1 & $12 \mathrm{i}$ & 1 & 0.1460 & 0.5282 & 0.0009 \\
$\mathrm{Bi} 2$ & $6 \mathrm{~h}$ & $\mathrm{~m}$ & 0.1766 & 0.2407 & $1 / 4$ \\
$\mathrm{U}$ & $6 \mathrm{~h}$ & $\mathrm{~m}$ & 0.3410 & 0.0917 & $1 / 4$ \\
$\mathrm{Ti}$ & $2 \mathrm{~b}$ & -3 & 0 & 0 & 0 \\
\hline
\end{tabular}

\section{Magnetic properties}

\subsection{Magnetic susceptibility}

Figures 3(a) and 3(b) shows the temperature $T$ dependence of the magnetic susceptibility $\chi(T)$ and magnetization curve $M(H)$ of $\mathrm{U}_{3} \mathrm{TiBi}_{9}$ for magnetic field $(H)$ parallel and perpendicular to the c-axis. $\chi(T)$ follows roughly as the Curie-Weiss law above $100 \mathrm{~K}$ to $300 \mathrm{~K}$ as shown in the inset of the Figure 3(a). The paramagnetic effective moment $3.5 \mu_{\mathrm{B}} / \mathrm{U}$ corresponds to the free uranium ion value, but there is $50 \%$ ambiguity because of the measurement using a very small single crystal. $\chi(T)$ shows large anisotropy, $\chi(T)$ perpendicular to the $\mathrm{c}$-axis is several times larger than that of the $\mathrm{c}$ axis. It is suggested that the easy axis of magnetization is perpendicular to the $\mathrm{c}$ axis.

$\chi(T)$ of the both axes show a peak at $31.5 \mathrm{~K}$ and decrease with decreasing temperature, especially the distinct decrease was observed on $\chi(T)$ of the easy axis. This anomaly does not show a hysteresis behavior within the measurement accuracy. It is considered that this anomaly is attributed to the antiferromagnetic ordering. The magnetization curves of the whole data exhibit linear $H$ dependence up to $10 \mathrm{kOe}$. We cannot observed any metamagnetic behavior at least until 10 $\mathrm{kOe}$. 

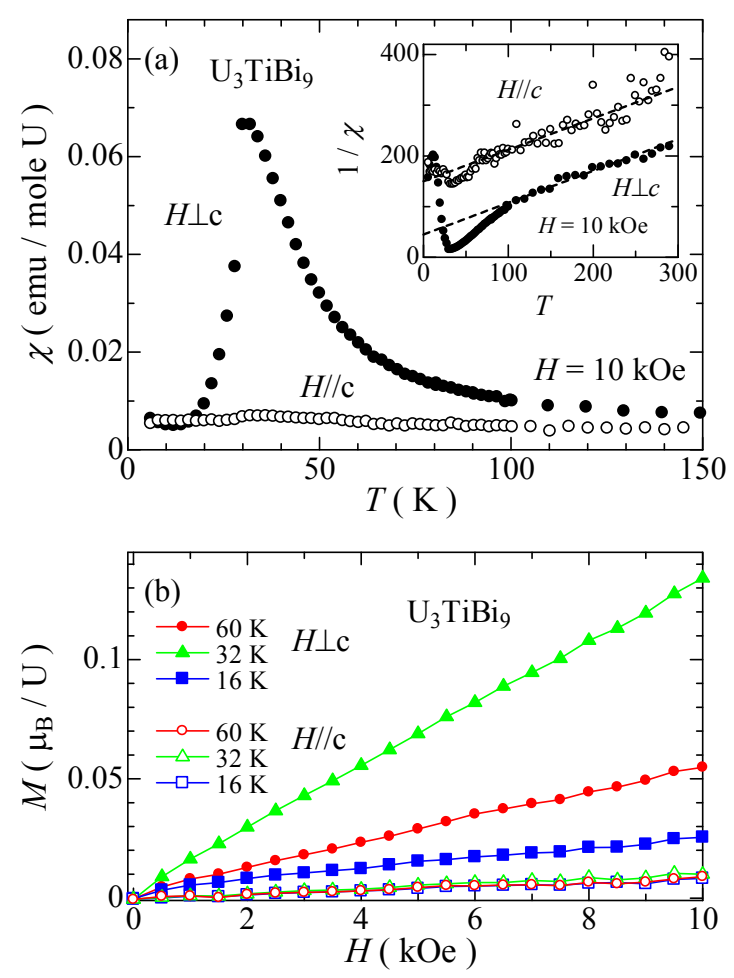

Figure 3. Temperature dependence of the magnetic susceptibility (a) and magnetization curve (b) of $\mathrm{U}_{3} \mathrm{TiBi}_{9}$. A static magnetic field were applied parallel and perpendicular to the c-axis. The inset of the (a) shows the $1 / \chi$ vs. $T$ plot.

\subsection{Electrical resistivity}

The $T$ dependences of electrical resistivity $\rho(T)$ and $T$ derivative of the electrical resistivity $\partial \rho / \partial T(T)$ on

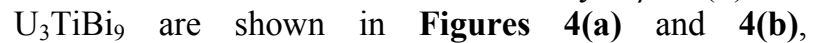
respectively. With decreasing temperature from $300 \mathrm{~K}$ to $80 \mathrm{~K}, \rho(T)$ gradually increases and then it rapidly decreases below $40 \mathrm{~K}$ after showing the flat $T$ dependence. The antiferromagnetic phase transition is observed as a small but clear kink at $31.5 \mathrm{~K}$. At low temperature, $\rho(T)$ finally approaches to a constant value.

The electrical resistivity as a function of $T^{2}$ as shown in the inset of the Figure 4(a) shows the linear dependence below $8 \mathrm{~K}$. A least square fit of the data to the expression $\rho(T)=\rho_{0}+A T^{2}$ gave $\rho_{0}=8.9 \mu \Omega \cdot \mathrm{cm}$ and $A=0.224 \mu \Omega \cdot \mathrm{cm} / \mathrm{K}^{2}$. The residual resistivity ratio $\rho_{300 \mathrm{~K}} / \rho_{0}$ is 29 , indicating a good quality sample. The large $A$ coefficient $0.224 \mu \Omega \cdot \mathrm{cm} / \mathrm{K}^{2}$ indicates a strong electron correlation, which is consistent with the Sommerfeld coefficient $\gamma=150 \mathrm{~mJ} /\left(\mathrm{K}^{2} \cdot \mathrm{mol}\right)$ according to the Kadowaki-Woods relation. For an accurate estimate of the $\gamma$ value, we should measure a specific heat of the $\mathrm{U}_{3} \mathrm{TiBi}_{9}$. It may be noted that a broad anomaly was observed around $16.5 \mathrm{~K}$ on the $\partial \rho / \partial T(T)$, where is lower than the magnetic transition temperature, although we do not have enough data about the broad anomaly to discuss it at present.

The antiferromagnetic transition temperature on $\rho(T)$ agrees with that on $\chi(T)$. The electrical resistivity of $\mathrm{U}_{3} \mathrm{TiBi}_{9}$ increases approximately as $\log T$ with

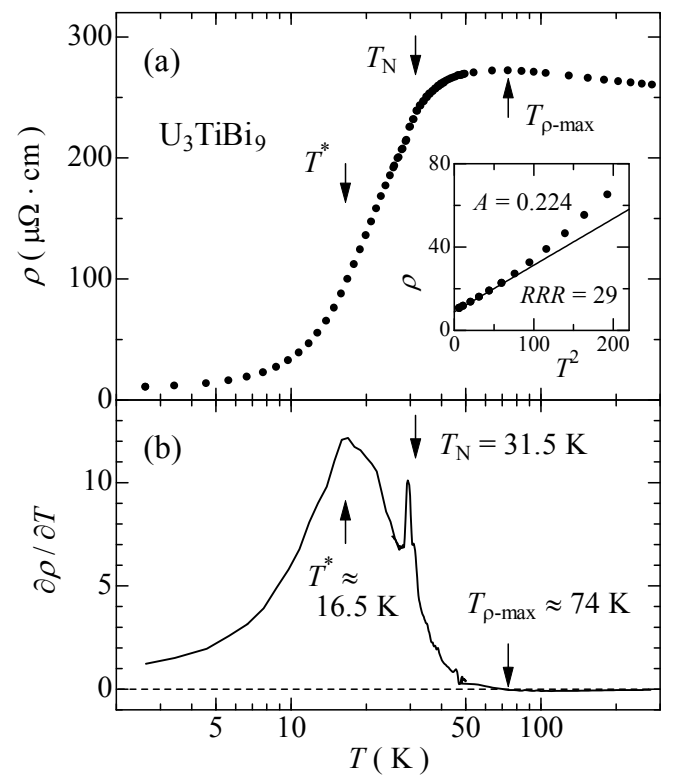

Figure 4. Temperature dependences of the electrical resistivity (a) and temperature derivative of the electrical resistivity (b) of $\mathrm{U}_{3} \mathrm{TiBi}_{9}$. The inset shows the electrical resistivity as a function of $T$ square. The current was applied along the c-axis.

decreasing $T$, this behavior well appears on the typical antiferromagnetic heavy fermion U-compounds such as $\mathrm{UPd}_{2} \mathrm{Al}_{3}[2]$. The cause of the $\log T$ dependence and the small anomaly at $T_{\mathrm{N}}$ on $\rho(T)$ is considered to be a competition between Kondo effect and RKKY exchange interaction in this system. However, the distinct decrease on $\chi(T)$ at $T_{\mathrm{N}}$ was observed, in spite of the small kink at $T_{\mathrm{N}}$ on $\rho(T)$. We need more future studies to discuss the ground state of this system.

\section{Conclusion}

We have found the U-Ti-Bi intermetallic compound $\mathrm{U}_{3} \mathrm{TiBi}_{9}$ and clarified that it crystallizes in the $P 6_{3} / \mathrm{m}$ hexagonal structure. The magnetic susceptibility of $\mathrm{U}_{3} \mathrm{TiBi}_{9}$ seems to follow the Curie-Weiss law at high temperature. This new uranium compound shows the antiferromagnetic ordering at $31.5 \mathrm{~K}$ and the Kondo behavior at least on the temperature dependence of the electrical resistivity.

\section{Acknowledgements}

We thank T. Mutou, K. Miyoshi, S. Nishigori, K. Fujiwara, N. K. Sato for valuable discussions and E. Yamamoto, K. Shirasaki for the equipment and technical support. This work was supported by JSPS KAKENHI(No. 16K05450), and carried out in the joint research in Institute for Materials Research, Tohoku University. 


\section{References}

[1] H.R. Ott, H. Rudigier, Z. Fisk and J.L. Smith, $\mathrm{UBe}_{13}$ : An Unconventional Actinide Superconductor, Phys. Rev. Lett. 50 (1983), pp. 15951598.

[2] C. Geibel, C. Schank, S. Thies, H. Kitazawa, C. D. Bredl, A. Böhm, M. Rau, A. Grauel, R. Caspary, R. Helfrich, U. Ahlheim, G. Weber and F. Steglieh, Heavy-fermion superconductivity at $=2 \mathrm{~K}$ in the antiferromagnet $\mathrm{UPd}_{2} \mathrm{Al}_{3}$, Z. Phys. B 84 (1991), pp. $1-2$.

[3] S.S. Saxena, P. Agarwal, K. Ahilan, F.M. Grosche, R.K.W. Haselwimmer, M.J. Steiner, E. Pugh, I.R. Walker, S.R. Julian, P. Monthoux, G.G. Lonzarich, A. Huxley, I. Sheikin, D. Braithwaite and J. Flouquet, Superconductivity on the border of itinerant-electron ferromagnetism in $\mathrm{UGe}_{2}$, Nature
406, (2000), pp. 587-592.

[4] S.H.D. Moore, L. Deakin, M.J. Ferguson and A. Mar, Physical properties and bonding in $R E_{3} \mathrm{TiSb}_{5}$ $(R E=\mathrm{La}, \mathrm{Ce}, \mathrm{Pr}, \mathrm{Nd}, \mathrm{Sm})$, Chem. Mater. 14 (2002), pp. 4867-4873.

[5] A. Mar, O. Tougait, M. Potel and H. Noël, Anisotropic transport and magnetic properties of ternary uranium antimonides $\mathrm{U}_{3} \mathrm{ScSb}_{5}$ and $\mathrm{U}_{3} \mathrm{TiSb}_{5}$, Chem. Mater. 18 (2006), pp. 4533-4540.

[6] G. Motoyama, M. Sezaki, J. Gouchi, K. Miyoshi, S. Nishigori, T. Mutou, K. Fujiwara and Y. Uwatoko, Magnetic properties of new antiferromagnetic heavy-fermion compounds $\mathrm{Ce}_{3} \mathrm{TiBi}_{5}$ and $\mathrm{CeTi}_{3} \mathrm{Bi}_{4}$, Proc. SCES- 2017, Physica B, (2017) in press.

[7] G.M. Sheldrick, A short history of SHELX, Acta Cryst. A64, (2008), pp. 112-122. 